\title{
A Capacitated Vehicle Routing Problem considering Satisfaction Level of Customers: A Genetic Algorithm
}

\author{
Mahnaz Naghshnilchi ${ }^{1, *}$
}

\begin{abstract}
Capacitated vehicle routing problem (CVRP) is one of the most well-known and applicable issues in the field of transportation. It has been proved to be an NP-Complete problem. To this end, it is needed to develop a high-performance algorithm to solve the problem, particularly in large scales. This paper develops a novel mathematical model for the CVRP considering the satisfaction level of demand nodes. Then, the proposed model is validated using a numerical example and sensitivity analyses that are implemented by CPLEX solver/GAMS software. To solve the problem efficiently, a Genetic Algorithm (GA) is designed and implemented. The obtained results demonstrate that the proposed GA can yield high-quality solutions compared to exact solutions.
\end{abstract}

Keywords: Vehicle routing problem, Genetic algorithm, Sensitivity analysis, Satisfaction level.

Manuscript was received on 14/03/2019, revised on 24/08/2019 and accepted for publication on 02/10/2019.

\section{Introduction}

Since the distribution of products accounts for an average of about $20 \%$ of the total cost of production, improving the efficiency of the transport of goods saves much on their cost and competition in the regional economy. Most product distribution issues can be considered as a vehicle routing problem (VRP), which is the generalization of the traveling salesman problem (TSP). One of the important issues in this field is the combination of many efficient methods to optimally solve it.

The efficient routing issue is economically important for both private and public sectors. VRP has been taken into account as a broad field of study, which was first introduced by Dantzig and Ramser [1]. The classic VRP model includes a set of customers and a set of homogenous vehicles such that each customer demands for products and is served only by a vehicle. The main objective is to minimize the total distance traveled by vehicles [2]. Due to the limitations of the real world, there are some extensions to the classic model. Capacitated vehicle routing problem (CVRP) is known as the most common transportation management problem in food, fuel and retail goods distributors that is studied in this research [3].

A review of the literature, including fleet composition issues and routing in road and sea transportation, was presented by Hoff et al. [4]. Motoya-Torres et al. [5] reviewed the literature on fleet and routing size, and the development of these issues along with future research directions and challenges. Most of the researchers tried to develop appropriate solution techniques to solve the CVRP.

A good update of different heuristic approaches can be found in Van Breedam [6]. Some important traditional studies about heuristic methods are Gaskell [7], Golden, et al. [3] and Bodin

\footnotetext{
* Corresponding Author.

${ }^{1}$ Department of Industrial Engineering, University of Isfahan, Isfahan, Iran, Email: mahnaz.naghsh2@gmail.com,
} 
and Berman [8]. The most remarkable reference in the VRP is the book published by Toth and Vigo [9] which presents a proper list of high-performance algorithms to solve the CVRP. Recently, a comprehensive review of meta-heuristic algorithms for solving the CVRP and other variants of VRP was conducted by Elshaer and Awad [10]. The literature of CVRP is rich in different metaheuristic algorithms such as simulated annealing (SA) [11], genetic algorithm (GA) [12], tabu search (TS) algorithm [13] and variable neighborhood search (VNS) algorithm [14]. On the other hand, other researchers have worked on other variants of routing problems that can be found in [1520].

In this study, in order to bring the CVRP closer to real conditions, the satisfaction level of demand nodes is considered in the problem. Therefore, the objective function tries to minimize the unmet demand besides the minimization of total traveling cost. Moreover, the proposed model seeks to use the minimum number of vehicles. To validate the proposed model, a numerical example is investigated using CPLEX solver/GAMS software. Then, an efficient GA is developed to deal with large-sized problems.

The remaining sections are organized as follows. Section 2 describes the main problem and presents the proposed mathematical model. Section 3 validates the proposed model and Section 4 introduces the applied GA. The numerical results and analysis is given in Section 5. Finally, the concluding remarks and future research directions are given in Section 6.

\section{Problem Description}

Consider a transportation network including a depot and a set of demand nodes (customers). This network can be described using a graph where the arcs are roads and vertices are junctions between them. Furthermore, there is a fleet of heterogeneous vehicles to fulfill the demand of customers. The aim is to find the optimal set of routes for the fleet where the total cost is minimized.

There are several objectives in this regard: minimizing the total number of used vehicles, maximizing the satisfaction rate of customers by meeting the demand for different products, or minimizing total unmet demand. Here, we consider these objective functions as a single objective in terms of the economic aspect.

Now, the main assumptions of the model are as follows:

- There are a number of demand nodes in this problem, whose demand is fulfilled through a single depot.

- There is a fleet of heterogeneous vehicles with limited capacities and different usage costs.

- Suppliers are unlimited.

- Demand of customers is given and deterministic.

- Vehicles start moving from the depot and pass through the nodes, then return to the depot.

- A particular product is considered to be delivered within the network.

- Satisfaction level of customers is directly dependent to the percentage of fulfilled demand.

The indexes, parameters and variables used in the model are as follows:

\section{Indexes}

$i, j, f \quad$ Index of nodes

$k \quad$ Index of vehicles

\section{Parameters}


Cap $^{k} \quad$ Capacity of vehicle $k$

$d_{i} \quad$ Demand of node $i$

$c_{i j} \quad$ Distance between nodes $i$ and $j$

$\gamma \quad$ Conversion coefficient of distance to cost

pen Penalty cost imposed by a unit of unmet demand

$f c_{k} \quad$ Usage cost of vehicle $k$

bigM An optional large number

\section{Variables}

$x_{i j}^{k} \quad$ A binary variable indicating whether arc $(i, j)$ is traversed by vehicle $k$ or not

$\alpha_{i}^{k} \quad$ Percentage of demand fulfilled by vehicle $k$ at node $i$

$D_{i} \quad$ Amount of unmet demand at node $i$

$y_{k} \quad$ A binary variable indicating whether vehicle $k$ is used or not

$u_{i}^{k} \quad$ An auxiliary variable related to node $i$ and vehicle $k$ to eliminate sub-tours

Now, the proposed mathematical model is given as follows:

minimize $\mathrm{Z}=\gamma\left(\sum_{j} \sum_{k} c_{i j} x_{i j}^{k}\right)+\sum_{k} f c_{k} y_{k}+\sum_{i}$ pen $D_{i}$

s.t. $\quad \sum_{i} x_{i j}^{k} \leq y_{k} \quad \forall j=2, \ldots, N, k$

$\sum_{i} x_{i f}^{k}-\sum_{j} x_{f j}^{k}=0 \quad \forall f, k$

$\sum_{j} x_{1 j}^{k}=y_{k} \quad \forall k$

$u_{i}^{k}+1 \leq u_{j}^{k}+\operatorname{big} M\left(1-x_{i j}^{k}\right) \quad \forall i \neq 1, j \neq 1, k$

$u_{1}^{k}=0 \quad \forall k$

$\sum_{k} x_{i i}^{k}=0 \quad \forall i$

$\sum_{i>1} \alpha_{i}^{k} d_{i} \leq \operatorname{Cap}^{k} \quad \forall k$

$\alpha_{i}^{k} \leq \sum_{j} x_{i j}^{k} \quad \forall i \neq 1, k$

$\alpha_{i}^{k} \leq y_{k} \quad \forall i \neq 1, k$

$D_{i}=\left(d_{i}-\sum_{k} \alpha_{i}^{k} d_{i}\right) \quad \forall i \neq 1$

$x_{i j}^{k} \in\{0,1\} \quad \forall i, j, k$

$u_{i}^{k}, D_{i}, \alpha_{i}^{k} \geq 0 \quad \forall i, k$ 
Objective function (1) represents the total cost minimization including traveling cost, vehicles' usage cost, and penalty cost of unmet demands. Eq. (2) indicates that if a vehicle is used, it can travel between two distinct nodes. Eq. (3) ensures that each vehicle must exit any node that has been already entered. Eq. (4) expresses that any vehicle used must start its trip from the depot (assuming the depot is node 1). Eqs. (5), (6) and (7) eliminate sub-tours. Eq. (8) represents the vehicle capacity limitation. Eq. (9) simultaneously ensures that each satisfaction rate takes a value between 0 and 1, as well as forcing it to take value only if the vehicle arrives at the relevant node. Eq. (10) shows that demand nodes receive the products only by vehicles that have been already used. Eq. (11) specifies the amount of cumulative demand that has not been fulfilled yet. Eqs. (12) and (13) show the types of variables.

\section{Model validation}

In this section, the model is solved using CPLEX solver/GAMS software as an exact solution method [21-22]. Moreover, different conditions are generated and compared as sensitivity analyses. Accordingly, the following results are achieved.

- If we increase the capacity or the number of vehicles, the satisfaction rate $\alpha$ increases.

- Nodes with close distances often belong to a single tour.

- If the number of vehicles is high, the number of service tours increases.

- As the number of vehicles increases with the total fixed capacity, the objective function changes as follows:

\begin{tabular}{|c|c|c|c|c|c|}
\hline Number of vehicles & 1 & 2 & 3 & 4 & 5 \\
\hline Objective value & 367 & 1215 & 2042 & 2954 & 1828 \\
\hline
\end{tabular}

- As vehicle capacity increases, the total satisfaction rate increases exponentially. Its linear approximation is also shown in Figure 1.

- By increasing demand and maintaining vehicle capacity, the total satisfaction rate of customers decreases dramatically.

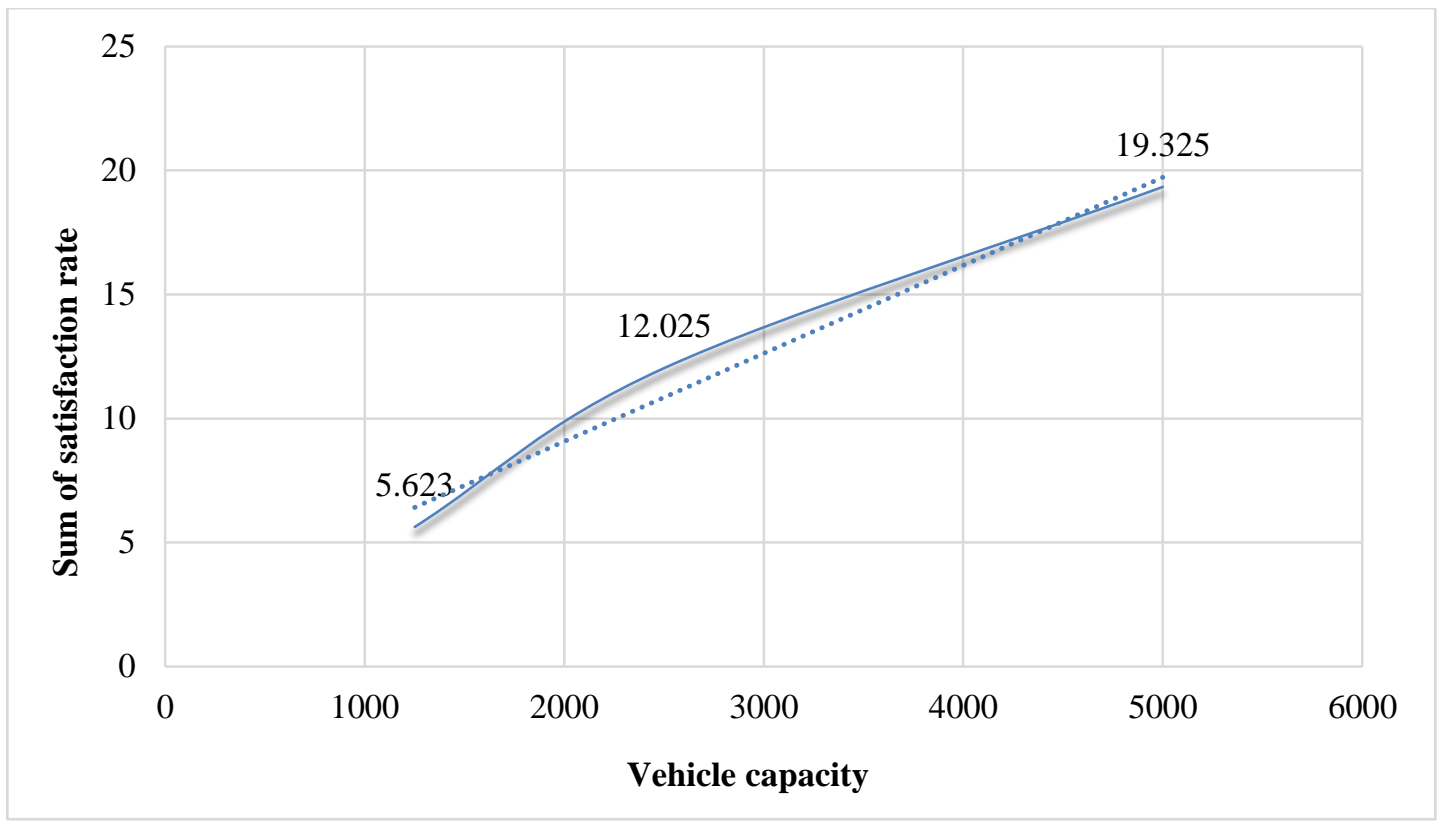

Figure 1. Sensitivity analysis of vehicle capacity. 


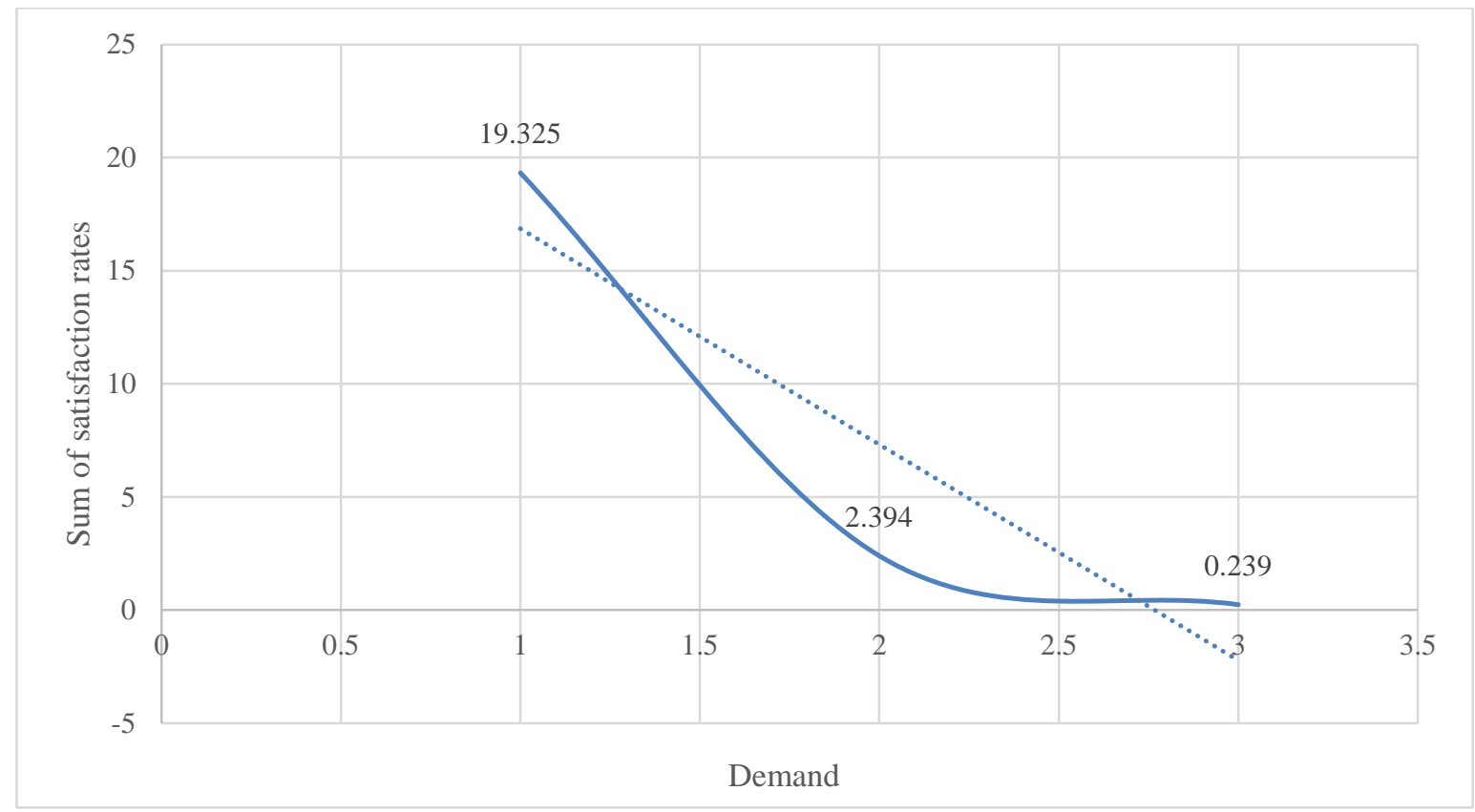

Figure 2. Sensitivity analysis of demand parameter.

\section{Genetic Algorithm}

GA is a well-known meta-heuristic algorithm inspired by the process of natural selection that is related to the larger class of evolutionary algorithms (EA). GAs are usually employed to generate high-quality solutions for optimization and search problems by regarding biological operators including mutation, crossover and selection [15]. It was introduced in 1960 based on the concept of Darwin's theory of evolution by John Holland [23].

The scope of GA is very wide and with the increasing progress of science and technology, the use of this method in optimizing and solving problems is greatly expanding. It is directly related to the subject of artificial intelligence. It can be called as a general search method that mimics the laws of natural biological evolution. The genetic algorithm applies the best survival law based on a series of solutions in the hope of getting better solutions. In each generation, with the help of a selection process commensurate with the value of the solutions and the regeneration of the selected solutions with the help of operators imitated by natural genetics, better approximations of the final solution are obtained. This process makes new generations more adaptable to the conditions of the problem.

\subsection{Chromosome-Solution Representation}

In this issue, there are a number of vehicles, each of which travels a certain route, and in its route carries a number of products with it and meets the demand of different customers. A string is defined for each vehicle as the chromosome. This string has two parts. In the first part, the served nodes of each device are specified in order, and in the second part, a percentage of the demand for delivered products to each node is determined. Figure 3 shows an example for this string. 


\begin{tabular}{|c|c|c|c|c|c|c|c|c|c|}
\hline $\mathbf{1}$ & $\mathbf{2}$ & $\mathbf{3}$ & $\mathbf{4}$ & $\mathbf{5}$ & $\mathbf{6}$ & $\mathbf{7}$ & $\mathbf{8}$ & $\mathbf{9}$ & $\mathbf{1 0}$ \\
\hline 1 & 0 & 0.3 & 0.4 & 0 & 0.2 & 0.6 & 0.5 & 0 & 0 \\
\hline 0 & 0 & 0.4 & 0.9 & 0 & 0.2 & 0.7 & 0.4 & 0 & 0 \\
\hline
\end{tabular}

Figure 3. Solution representation.

In the above field, it is assumed that there were 10 nodes in total. After starting the tour from the depot (node 1), the vehicle visits five nodes 8, 7, 6, 3 and 4. However, the order of the visited nodes can be determined by arranging the priority of their visit, which is shown in the first row of the string. In this example, after exiting the depot, the vehicle visits node 6 , then node 3 , node 4 , node 8 , and finally node 7 . In each node, a percentage of their demand is met, which is shown in the second row of the string. It should be noted that if a vehicle is not used, all the corresponding values in the string is set to zero

\subsection{Calculation of Objective Function-Fitness Value}

To calculate the objective function, first, the service route of each vehicle is determined, then the cost of the route of each vehicle is calculated. The sum of these costs for different vehicles determines the cost of routing. The amount of demand met from each node is then determined. If the total demand of a point is not met, the unmet demand of those nodes will be included in the second part of the function.

On the other hand, according to the defined string, the algorithm is not forced to observe the vehicle capacity limitation. The reason is to extend the search space of the algorithm. For this purpose, if the vehicle carries more than its capacity, a penalty cost related to the excess load is imposed on the objective function. The coefficient of this penalty is 10,000 units.

\subsection{Crossover and Mutation}

In order to apply a crossover between two different solutions, a one-point crossover method is used by selecting a string from each parent and dividing both strings into two parts based on a specific node. For more information, see [24]. Each child then inherits one part of the first parent string and one part of the other parent string.

Moreover, each chromosome in each generation with a probability of $50 \%$ is modified using a mutation operator. In the mutation process, a cell is selected from a chromosome and its value changes randomly.

\section{Numerical Results}

This section evaluates the performance of the GA used in this research compared to CPLEX solver/GAMS software. This algorithm was coded in MATLAB. Furthermore, 10 problems are considered to perform the comparisons.

To generate these problem instances, the demand of each node is uniformly distributed with a low bound of $1000 \mathrm{~kg}$ and a high bound of $2000 \mathrm{~kg}$. Each node is located in a two-dimensional space with a length of $150 \mathrm{~km}$ and a width of $150 \mathrm{~km}$, and the travel cost between the two nodes corresponds to the distance between the two nodes. The capacity of the vehicles is randomly considered to be between $8000 \mathrm{~kg}$ and $15000 \mathrm{~kg}$. Obviously, the capacity of the vehicles is chosen in such a way that the total capacity of the vehicles exceeds the total demand of the nodes. Other information on the problems is shown in Table 1. 
Table 1. Information of the problem instances.

\begin{tabular}{|c|c|c|c|}
\hline Problem & $\boldsymbol{n}$ & $\boldsymbol{k}$ & $\boldsymbol{J}$ \\
\hline PR1 & 6 & 2 & 30 \\
\hline PR2 & 10 & 2 & 30 \\
\hline PR3 & 20 & 3 & 25 \\
\hline PR4 & 30 & 5 & 20 \\
\hline PR5 & 40 & 10 & 20 \\
\hline PR6 & 50 & 12 & 15 \\
\hline PR7 & 70 & 15 & 15 \\
\hline PR8 & 100 & 20 & 10 \\
\hline PR9 & 150 & 30 & 10 \\
\hline PR10 & 200 & 40 & 5 \\
\hline
\end{tabular}

In the following, the obtained results by the solution methods are given in Table 2 .

Since GAMS software is very time-consuming to solve high-dimensional problems, it has a time limit of 3600 seconds or 1 hour. It should be noted that if solving the problem in GAMS software takes more than 1 hour, GAMS software will provide a justified (not necessarily optimal) answer after 1 hour and the program will be completed.

The following table summarizes the results of comparing GAMS software with the genetic algorithm

Table 2. Obtained numerical results.

\begin{tabular}{|c|c|c|c|c|c|}
\hline \multirow{2}{*}{ Problem } & \multicolumn{2}{|c|}{ CPLEX solver } & \multicolumn{2}{c|}{ GA } & \multirow{2}{*}{ Gap (\%) } \\
\cline { 2 - 5 } & $\begin{array}{c}\text { Objective } \\
\text { function }\end{array}$ & Run time (s) & $\begin{array}{c}\text { Objective } \\
\text { function }\end{array}$ & Run time (s) & \\
\hline PR1 & 8593.01 & 1.02 & 8624.79 & 18.19 & 0.37 \\
\hline PR2 & 12678.21 & 3.50 & 12784.50 & 21.60 & 0.84 \\
\hline PR3 & 22540.08 & 9.71 & 22718.07 & 34.95 & 0.79 \\
\hline PR4 & 39765.36 & 49.38 & 40174.58 & 49.70 & 1.03 \\
\hline PR5 & 66038.19 & 103.50 & 67121.02 & 58.92 & 1.64 \\
\hline PR6 & 103957.84 & 485.16 & 107013.34 & 79.02 & 2.94 \\
\hline PR7 & 259760.33 & 1672.20 & 268539.89 & 102.88 & 3.38 \\
\hline PR8 & 546375.05 & 2953.90 & 569268.11 & 129.73 & 4.19 \\
\hline PR9 & 896485.16 & 3600.00 & 933061.59 & 154.64 & 4.08 \\
\hline PR10 & - & 3600.00 & 1429201.01 & 188.10 & - \\
\hline Average & - & 1247.84 & - & 83.77 & 2.14 \\
\hline
\end{tabular}

As can be seen in Table 2, GAMS software was not able to find the optimal solution in the last problem in less than 1 hour, but for all problem instances, the proposed GA could solve the problem with acceptable gaps compared to CPLEX solver/GAMS software; i.e., the average gap of $2.14 \%$. Moreover, the average run time to solve the problem instances is 1247.84 and 83.77 seconds for CPLEX solver/GAMS software and GA, respectively.

To better understand the comparisons, Figures 4 and 5 represent the run time and objective function comparisons, respectively. 


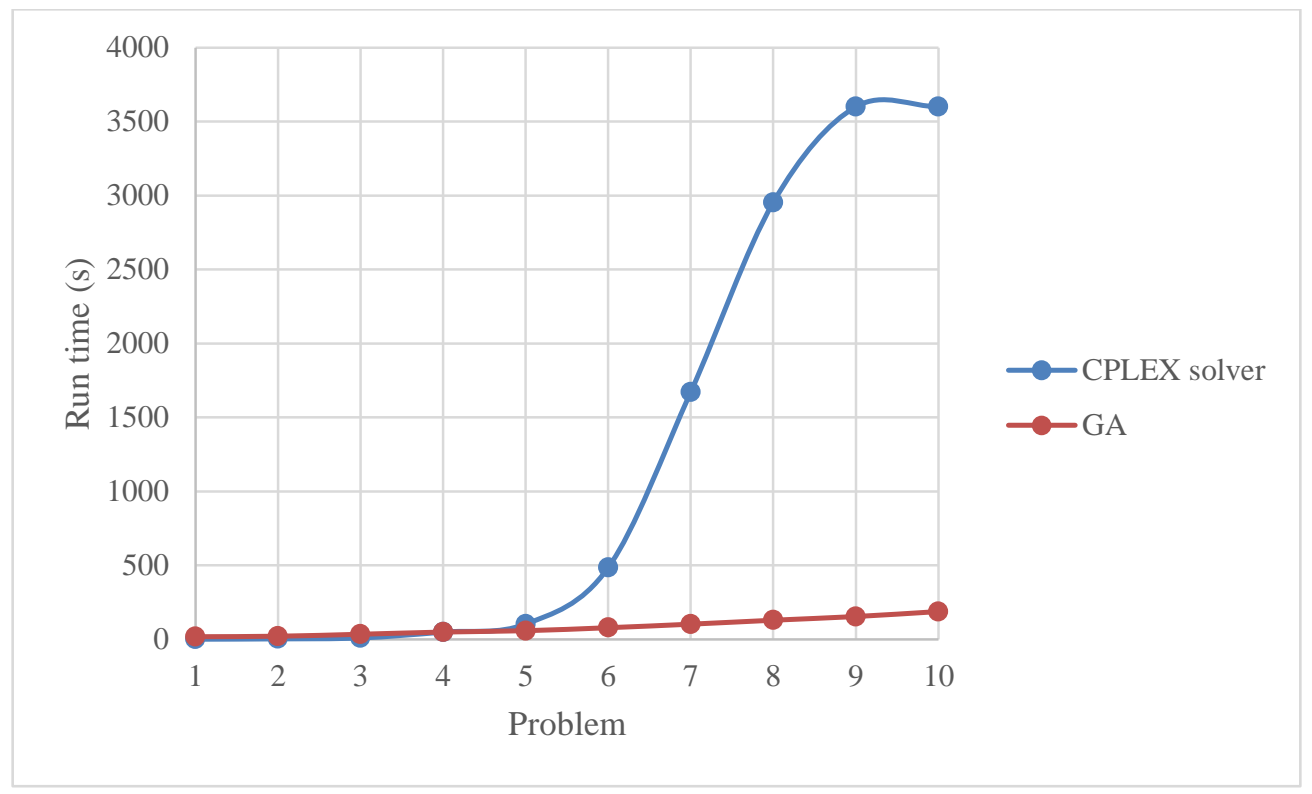

Figure 4. Run time comparison.

As can be seen, the run time values of CPLEX solver/GAMS software have an exponential increase, which grow significantly with the increase of the problem scale. However, in the proposed GA, the rate of increase in run time is relatively small.

Figure 5 depicts the comparison between the objective functions obtained by CPLEX solver/GAMS software and GA.

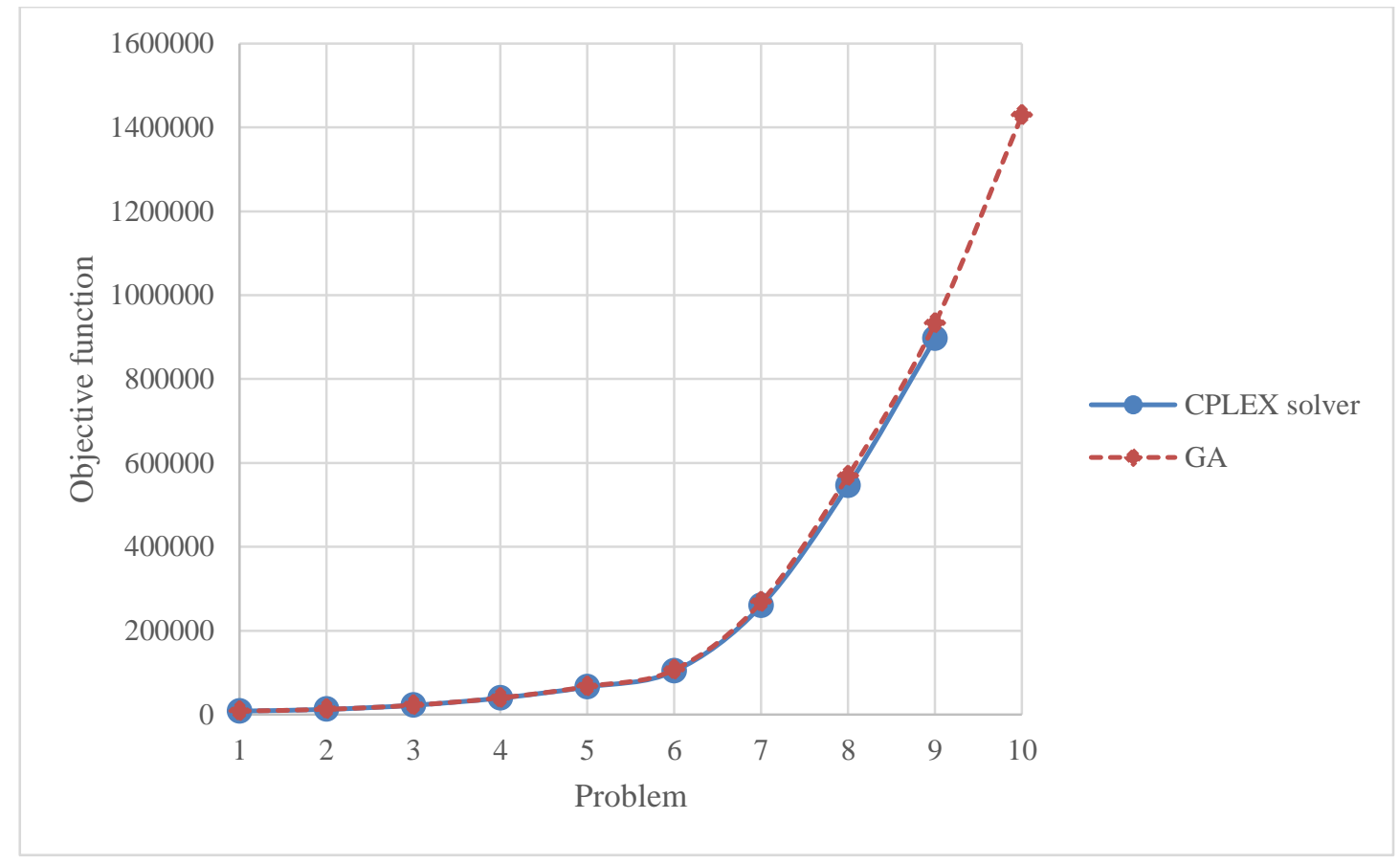

Figure 5. Objective function comparison. 
According to Figure 5, it can be found that the suggested GA can efficiently solve the problem instances close to CPLEX solver/GAMS software. Overall, it can be concluded that our GA is able to find high-quality solutions within a reasonable run time and can be used as an efficient solution tool.

\section{Conclusion and Outlook}

In this paper, a novel formulation was developed to formulate the CVRP considering customers' satisfaction level. The objective was to minimize the total cost including traveling cost, vehicles' usage cost and penalty cost of unmet demands. The proposed MILP model was then validated using a numerical example and sensitivity analyses by CPLEX solver/ GAMS software. Moreover, to solve the problem according to the real-world conditions, an efficient GA was proposed and applied to 10 different problems in small, medium and large scales. The obtained results demonstrated that the proposed algorithm is able to find high-quality solutions with an average gap and average run time of $2.14 \%$ and 83.77 seconds, respectively.

Since there are some major limitations in the study, future research directions can be designed accordingly. In the following, the most important recommendations are given:

1) Considering a time horizon to fulfill the demand of customers [25],

2) Extending the problem considering more real-world assumptions, such as time windows constraint [26],

3) Applying uncertainty techniques to study the uncertain nature of the parameters, such as fuzzy programming [27-28] and robust optimization [29-30],

4) Developing other algorithms to evaluate the performance of the proposed GA, such as runner root algorithm (RRA) [31], particle swarm optimization (PSO) algorithm [32] and cuckoo optimization algorithm (COA) [33].

5) Considering other objectives (e.g., pollution minimization [34]) and applying efficient multiobjective meta-heuristic algorithms, such as non-dominated sorting genetic algorithm III (NSGA-III) [35].

\section{Reference list:}

[1] Dantzig, G. B., \& Ramser, J. H. (1959). The truck dispatching problem. Management Science, 6(1), 80-91.

[2] Ombuki-Berman, B., \& Hanshar, F. T. (2009). Using genetic algorithms for multi-depot vehicle routing. In Bio-inspired algorithms for the vehicle routing problem (pp. 77-99). Springer, Berlin, Heidelberg.

[3] Golden, B., Assad, A., Levy, L., \& Gheysens, F. (1984). The fleet size and mix vehicle routing problem. Computers \& Operations Research, 11(1), 49-66.

[4] Hoff, A., Andersson, H., Christiansen, M., Hasle, G., \& Løkketangen, A. (2010). Industrial aspects and literature survey: Fleet composition and routing. Computers \& Operations Research, 37(12), 2041-2061.

[5] Montoya-Torres, J. R., Franco, J. L., Isaza, S. N., Jiménez, H. F., \& Herazo-Padilla, N. (2015). A literature review on the vehicle routing problem with multiple depots. Computers \& Industrial Engineering, 79, 115-129.

[6] Van Breedam, A. (2001). Comparing descent heuristics and metaheuristics for the vehicle routing problem. Computers \& Operations Research, 28(4), 289-315.

[7] Gaskell, T. J. (1967). Bases for vehicle fleet scheduling. Journal of the Operational Research Society, 18(3), 281-295.

[8] Bodin, L. D., \& Berman, L. (1979). Routing and scheduling of school buses by computer. Transportation Science, 13(2), 113-129.

[9] Toth, P., \& Vigo, D. (Eds.). (2002). The vehicle routing problem. Society for Industrial 
and Applied Mathematics.

[10] Elshaer, R., \& Awad, H. (2020). A taxonomic review of metaheuristic algorithms for solving the vehicle routing problem and its variants. Computers \& Industrial Engineering, $140,106242$.

[11] Wei, L., Zhang, Z., Zhang, D., \& Leung, S. C. (2018). A simulated annealing algorithm for the capacitated vehicle routing problem with two-dimensional loading constraints. European Journal of Operational Research, 265(3), 843-859.

[12] Kuo, R. J., Zulvia, F. E., \& Suryadi, K. (2012). Hybrid particle swarm optimization with genetic algorithm for solving capacitated vehicle routing problem with fuzzy demand-A case study on garbage collection system. Applied Mathematics and Computation, 219(5), 2574-2588.

[13] Tao, Y., \& Wang, F. (2015). An effective tabu search approach with improved loading algorithms for the 3L-CVRP. Computers \& Operations Research, 55, 127-140.

[14] Amous, M., Toumi, S., Jarboui, B., \& Eddaly, M. (2017). A variable neighborhood search algorithm for the capacitated vehicle routing problem. Electronic Notes in Discrete Mathematics, 58, 231-238.

[15] Tirkolaee, E. B., Hosseinabadi, A. A. R., Soltani, M., Sangaiah, A. K., \& Wang, J. (2018). A hybrid genetic algorithm for multi-trip green capacitated arc routing problem in the scope of urban services. Sustainability, 10(5), 1366.

[16] Tirkolaee, E. B., Mahdavi, I., \& Esfahani, M. M. S. (2018). A robust periodic capacitated arc routing problem for urban waste collection considering drivers and crew's working time. Waste Management, 76, 138-146.

[17] Tirkolaee, E. B., Alinaghian, M., Hosseinabadi, A. A. R., Sasi, M. B., \& Sangaiah, A. K. (2019). An improved ant colony optimization for the multi-trip Capacitated Arc Routing Problem. Computers \& Electrical Engineering, 77, 457-470.

[18] Babaee Tirkolaee, E., Abbasian, P., Soltani, M., \& Ghaffarian, S. A. (2019). Developing an applied algorithm for multi-trip vehicle routing problem with time windows in urban waste collection: A case study. Waste Management \& Research, 37(1_suppl), 4-13.

[19] Tirkolaee, E. B., Hadian, S., Weber, G. W., \& Mahdavi, I. (2020). A robust green trafficbased routing problem for perishable products distribution. Computational Intelligence, 36(1), 80-101.

[20] Tirkolaee, E. B., Goli, A., Bakhshi, M., \& Sangaiah, A. K. (2019). An Efficient Biography-Based Optimization Algorithm to Solve the Location Routing Problem With Intermediate Depots for Multiple Perishable Products. In Deep Learning and Parallel Computing Environment for Bioengineering Systems (pp. 189-205). Academic Press.

[21] Tirkolaee, E. B., Mardani, A., Dashtian, Z., Soltani, M., \& Weber, G. W. (2020). A novel hybrid method using fuzzy decision making and multi-objective programming for sustainable-reliable supplier selection in two-echelon supply chain design. Journal of Cleaner Production, 250, 119517.

[22] Tirkolaee, E. B., Mahdavi, I., Esfahani, M. M. S., \& Weber, G. W. (2020). A robust green location-allocation-inventory problem to design an urban waste management system under uncertainty. Waste Management, 102, 340-350.

[23] Cui, J., Fogarty, T. C., \& Gammack, J. G. (1993). Searching databases using parallel genetic algorithms on a transputer computing surface. Future Generation Computer Systems, 9(1), 33-40.

[24] Visutarrom, T., \& Chiang, T. C. (2019, June). An Evolutionary Algorithm with Heuristic Longest Cycle Crossover for Solving the Capacitated Vehicle Routing Problem. In 2019 IEEE Congress on Evolutionary Computation (CEC) (pp. 673-680). IEEE.

[25] Babaee Tirkolaee, E., Goli, A., Pahlevan, M., \& Malekalipour Kordestanizadeh, R. 
(2019). A robust bi-objective multi-trip periodic capacitated arc routing problem for urban waste collection using a multi-objective invasive weed optimization. Waste Management \& Research, 37(11), 1089-1101.

[26] Tirkolaee, E. B., Goli, A., Bakhsi, M., \& Mahdavi, I. (2017). A robust multi-trip vehicle routing problem of perishable products with intermediate depots and time windows. Numerical Algebra, Control \& Optimization, 7(4), 417-433.

[27] Tirkolaee, E. B., Goli, A., \& Weber, G. W. (2020). Fuzzy Mathematical Programming and Self-Adaptive Artificial Fish Swarm Algorithm for Just-in-Time Energy-Aware Flow Shop Scheduling Problem with Outsourcing Option. IEEE Transactions on Fuzzy Systems. doi: 10.1109/TFUZZ.2020.2998174

[28] Tirkolaee, E. B., Goli, A., \& Weber, G. W. (2019). Multi-objective aggregate production planning model considering overtime and outsourcing options under fuzzy seasonal demand. In Advances in manufacturing II (pp. 81-96). Springer, Cham.

[29] Golpîra, H., \& Tirkolaee, E. B. (2019). Stable maintenance tasks scheduling: A biobjective robust optimization model. Computers \& Industrial Engineering, 137, 106007.

[30] Sangaiah, A. K., Tirkolaee, E. B., Goli, A., \& Dehnavi-Arani, S. (2019). Robust optimization and mixed-integer linear programming model for LNG supply chain planning problem. Soft Computing, 1-21.

[31] Goli, A., Zare, H. K., Tavakkoli-Moghaddam, R., \& Sadeghieh, A. (2019). Hybrid artificial intelligence and robust optimization for a multi-objective product portfolio problem Case study: The dairy products industry. Computers \& Industrial Engineering, $137,106090$.

[32] Tirkolaee, E. B., Mahmoodkhani, J., Bourani, M. R., \& Tavakkoli-Moghaddam, R. (2019). A Self-Learning Particle Swarm Optimization for Robust Multi-Echelon Capacitated Location-Allocation-Inventory Problem. Journal of Advanced Manufacturing Systems, 18(04), 677-694.

[33] Goli, A., Aazami, A., \& Jabbarzadeh, A. (2018). Accelerated cuckoo optimization algorithm for capacitated vehicle routing problem in competitive conditions. International Journal of Artificial Intelligence, 16(1), 88-112.

[34] Elhedhli, S., \& Merrick, R. (2012). Green supply chain network design to reduce carbon emissions. Transportation Research Part D: Transport and Environment, 17(5), 370-379.

[35] Sangaiah, A. K., Goli, A., Tirkolaee, E. B., Ranjbar-Bourani, M., Pandey, H. M., \& Zhang, W. (2020). Big Data-Driven Cognitive Computing System for Optimization of Social Media Analytics. IEEE Access, 8, 82215-82226. 